\title{
Clinical Impact of Switching to Ceritinib After Severe AEs Related to Crizotinib/Alectinib in a Novel PTH2R-ALK Fusion Lung Adenocarcinoma: A Case Report
}

\author{
Gang Shen ${ }^{1, *}$ \\ Yinping $\mathrm{Du}^{\mathrm{l}}{ }^{\prime} *$ \\ Jifang Shen' \\ Junling Zhang $\mathbb{( D}^{2}$ \\ Xihua $\mathrm{Xia}^{2}$ \\ Mengli Huang $\mathbb{B}^{2}$ \\ Wenxiang Shen ${ }^{3}$ \\ 'Department of Radiology, The Affiliated \\ Kunshan Hospital of Jiangsu University, \\ Kunshan, People's Republic of China; \\ ${ }^{2}$ The Medical Department, 3D Medicines, \\ Inc., Shanghai, People's Republic of China; \\ ${ }^{3}$ Department of Oncology, The Affiliated \\ Kunshan Hospital of Jiangsu University, \\ Kunshan, People's Republic of China \\ *These authors contributed equally to \\ this work
}

Correspondence: Wenxiang Shen Department of Oncology, The Affiliated Kunshan Hospital of Jiangsu University, 9I West Qianjin Road, Kunshan, Jiangsu Province, 215300, People's Republic of China

Email shenwenxiang329@I63.com

\begin{abstract}
Lung cancer is still the leading cause of morbidity and mortality by cancer among men, according to the latest epidemiological data in China. Anaplastic lymphoma kinase $(A L K)$ rearrangements act as key oncogenic drivers of non-small cell lung cancer (NSCLC) and have been identified in 5-6\% of NSCLC. Although ALK inhibitors (ALK-TKIs) were proven to be more effective than chemotherapy in ALK-positive NSCLC patients and the safety profile of these drugs was favorable, novel ALK fusions NSCLC might discontinue or switch treatment because of adverse events (AEs) have rarely previously been reported. Here, we describe a male patient with stage IV lung adenocarcinoma who carried a novel PTH2R-ALK fusion identified by next-generation sequencing (NGS). The patient first took crizotinib but switched to alectinib due to gastrointestinal AEs. Although alectinib remained effective on tumors, ceritinib (450 mg) was replaced after the AEs of hyperbilirubinemia occurred. After reducing the dose to $300 \mathrm{mg}$, the diarrhea AEs caused by ceritinib were effectively relieved, and the patient obtained sustained clinical benefit with progression-free survival nearly 12 months. Our findings offer valuable information for the safety management of NSCLC patients with a novel PTH2R-ALK fusion treated by ALK-TKIs.
\end{abstract}

Keywords: $P T H 2 R-A L K$, non-small cell lung cancer, ceritinib, adverse events

\section{Introduction}

Non-small-cell lung cancer (NSCLC) accounts for approximately $80-85 \%$ of lung cancers and is the leading cause of cancer-related deaths in both men and women in China. ${ }^{1}$ The anaplastic lymphoma kinase $(A L K)$ gene encodes a transmembrane tyrosine kinase receptor, which consists of an extracellular domain, a transmembrane segment, and a cytoplasmic receptor kinase domain. ${ }^{2} A L K$ rearrangements are common driving mutations in the development of NSCLC and are found in approximately $5 \%$ of NSCLC cases. ${ }^{3}$ Since the $E M L 4-A L K$ rearrangement was first identified in NSCLC in $2007,{ }^{4}$ several $A L K$ partner genes have been reported such as $K I F 5 B, K L C 1$, PRKAR1A, GCC2. ${ }^{5}$ With rapid development of next-generation sequencing (NGS) technology, a variety of novel $A L K$ fusion genes have been discovered, which enrich the $A L K$ fusion spectrum.

The $A L K$ inhibitor crizotinib has become the standard therapy in advanced $A L K$ positive NSCLC since 2011. ${ }^{6}$ Subsequently, second-generation ALK-TKIs, alectinib and ceritinib were also proved to have good antitumor activity. ${ }^{7}$ However, different 
forms of $A L K$ rearrangements exhibit varied responses and treatment-related adverse events (AEs) to ALK-TKI therapy. ${ }^{8}$ It is reported that about $60-80 \%$ of the patients adjust the dose, interrupt or delay treatment due to toxic side effects. ${ }^{9}$ Therefore, it is important and necessary to select a treatment with acceptable toxicological properties and less impact on patients' quality of life. The most common AEs of these ALK-TKIs include gastrointestinal effects, fatigue, rash, and hepatotoxicity. ${ }^{10}$ In a pooled safety analyses of ALK-TKIs, including clinical trials receiving crizotinib, alectinib or ceritinib, the overall frequency of grade $\geq 3$ AEs was about $22.9-49.7 \%{ }^{11}$ Thus, the management of AEs in $A L K$-positive NSCLC receiving ALK-TKIs should be taken into account.

Herein, we describe that a lung adenocarcinoma patient carrying a novel $P T H 2 R-A L K$ fusion who received reduced doses of ceritinib after discontinuation of crizotinib and alectinib, with clinical benefit and good tolerability.

\section{Case Presentation}

An 83-year-old man was admitted to the Respiratory Department of the Affiliated Kunshan Hospital of Jiangsu University on October 30, 2019, due to cough and expectoration accompanying hemoptysis for 1 week. The patient had a history of tuberculosis for more than 30 years and chronic bronchitis and emphysema for 10 years. Chest computed tomography (CT) revealed an irregular mass $(5.3 \times 2.6 \mathrm{~cm})$ in the upper lobe of his left lung (Figure 1A). Furthermore, positron emission tomography/computed tomography (PET/CT) showed multiple lymph node and bone metastases (Figure 1B). The patient underwent a transthoracic core needle biopsy of the lung mass. Histological staining of lung tissue showed obvious tumor cell characteristics with adenoid and stratified structures and visible nuclear division
(Figure 1C). Immunohistochemistry analysis revealed positivity for thyroid transcription factor 1(TTF-1), CK7, SPA, napsin-A and Ki-67 (about 10\%), but negativity for villin, P63 and P40. Taken together, he was diagnosed with stage IV (T4aN2M1) invasive lung adenocarcinoma.

On December 6, 2019, a novel PTH2R-ALK fusion was identified in the patient's tumor tissue by NGS (Figure 2A), which was composed of exon 1 of $P T H 2 R$ (parathyroid hormone second receptor) gene and exon 20-29 of $A L K$ gene (Figure 2B). Fluorescence in situ hybridization further confirmed the $A L K$ positive (Figure 2C). It contained the entire kinase domain of $A L K$ and may activate $A L K$ signaling to play a role in tumor genesis and development. Based on the results of NGS test, the patient started oral crizotinib $250 \mathrm{mg}$ BID from December 12, 2019. After taking crizotinib for 10 days, the patient developed mild nausea without vomiting. Reexamination of liver function revealed that the level of alanine transaminase (ALT) and aspartate transaminase (AST) was 3-4 times higher than baseline (Table 1), suggesting mild liver function impairment. Thus, the patient was treated with polyene phosphatidylcholine for liver protection. The irregular mass shadow of the upper lobe of the left lung shrank significantly to $1.47 \times 0.89 \mathrm{~cm}$ when reexamined by CT on January 18, 2020, indicating that this patient achieved a partial response.

However, considering the intolerable hepatotoxicity, the patient switched to take alectinib $1200 \mathrm{mg}$ BID from January 20, 2020. After 1 week, the clinician reduced the dosage to $600 \mathrm{mg}$ BID, but the patient still had swelling in his legs and felt fatigued. His uncomfortable symptoms were slightly eased after symptomatic treatment of spironolactone. Carcinoembryonic antigen decreased obviously after treatment for a month. However, the total bilirubin of this patient reached up to three times of reference range
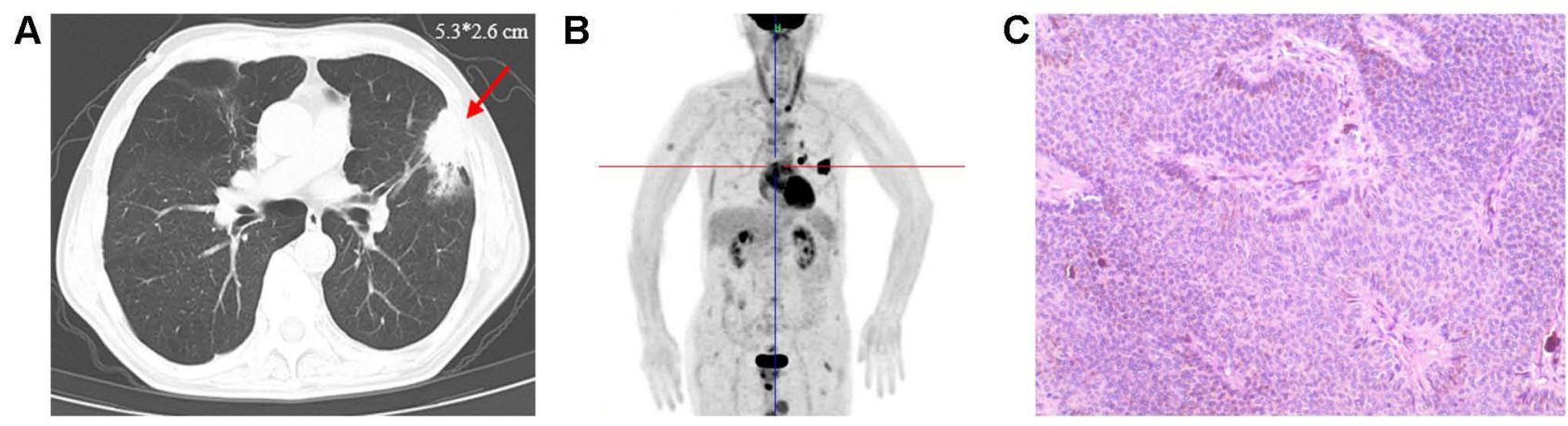

Figure I Diagnosis results by CT scan and pathology. (A) CT diagnosis of lung tissue. (B) Image of PET/CT scan. (C) Pathological diagnosis result (I00× magnification). 


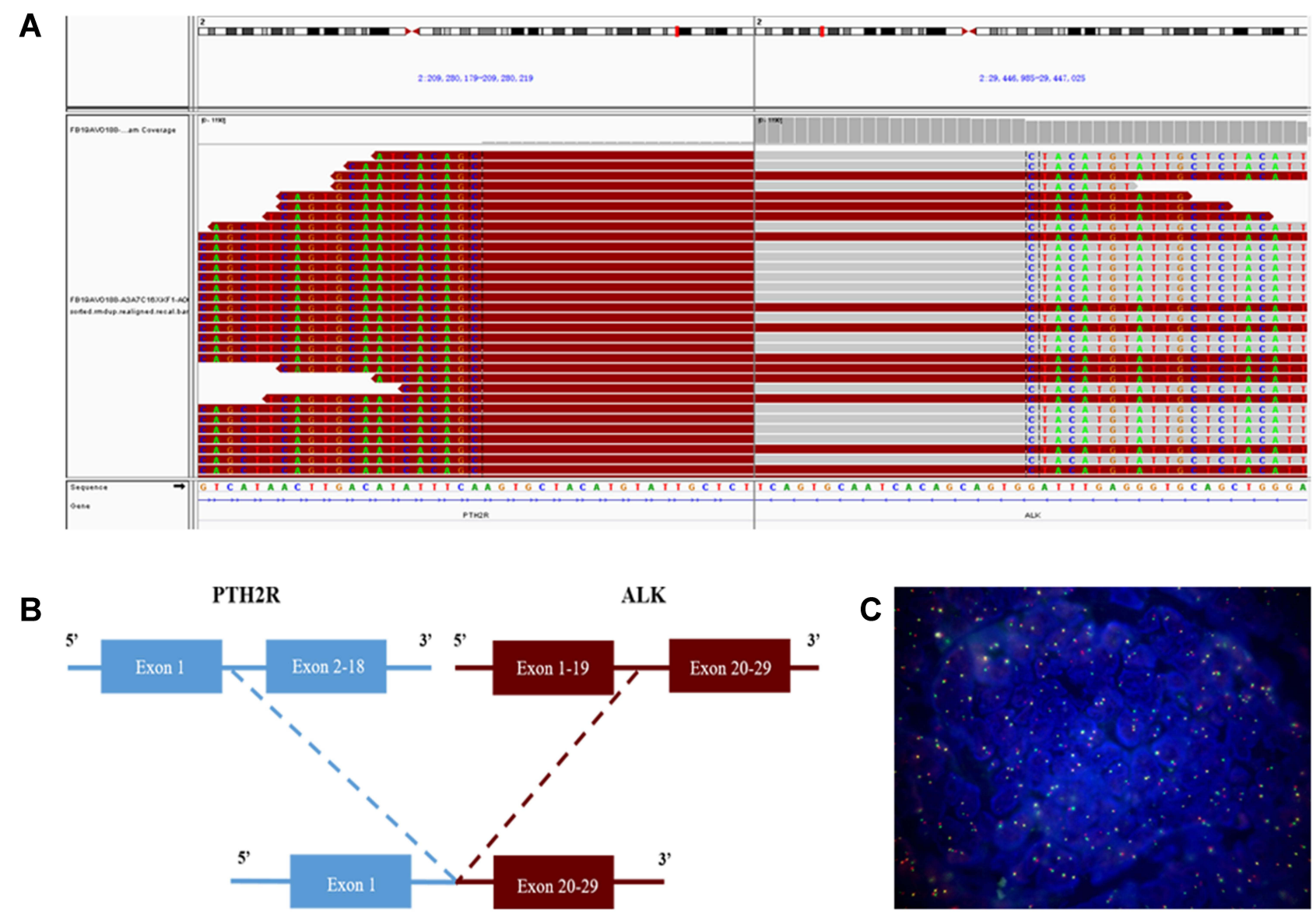

Figure 2 NGS and FISH findings for the primary lung tumor tissue sample. (A) NGS results showed breakpoint of PTH2R-ALK fusion. (B) A novel intergenic region between PTH2R exon I and ALK exon 20-29 fusion variant was identified. (C) A split signal was observed with a frequency of $25 \%$ in the FISH image (400x magnification).

(Table 1). Furthermore, he did not respond to symptomatic treatment of liver protection. On June 20, 2020, the patient started ceritinib orally $450 \mathrm{mg}$ QD with food. He suffered from diarrhea and antidiarrheal drugs were ineffective. Then, the clinician reduced the dosage of ceritinib to $300 \mathrm{mg}$ QD. During treatment with dose reduction of ceritinib, the patient's blood routine tests and liver function tests

Table I Liver Function Tests Before and After ALK-TKIs Treatment

\begin{tabular}{|l|c|c|c|c|}
\hline & $\begin{array}{c}\text { Before } \\
\text { Treatment }\end{array}$ & $\begin{array}{c}\text { After } \\
\text { Crizotinib }\end{array}$ & $\begin{array}{c}\text { After } \\
\text { Alectinib }\end{array}$ & $\begin{array}{c}\text { After } \\
\text { Ceritinib }\end{array}$ \\
\hline ALT (U/L) & 14.0 & 205.0 & 16.0 & 16.0 \\
AST (U/L) & 16.0 & 114.0 & 30.0 & 30.0 \\
$\gamma-$ GT (U/L) & 18.0 & 69.0 & 13.0 & 30.0 \\
ALP (U/L) & 71.0 & 105.0 & 154.0 & 98.0 \\
LDH (U/L) & 150.0 & 389.0 & 343.0 & 233.0 \\
TBIL ( $\mu \mathrm{mol} / \mathrm{L})$ & 29.7 & 7.1 & 62.7 & 22.9 \\
\hline
\end{tabular}

Abbreviations: ALT, alanine transaminase; AST, aspartate transaminase; $\gamma-\mathrm{GT}$, $\gamma$-glutamyl transpeptidase; ALP, alkaline phosphatase; LDH, lactate dehydrogenase; TBIL, total bilirubin. were normal, and chest CT scan demonstrated stable disease. The patients were followed up several times, with the last follow-up on June 13, 2021. The tumor lesion in the left lung remained stable and PFS was nearly 12 months.

\section{Discussion}

In this case, a novel fusion form of $A L K$ rearrangement (PTH2R-ALK) consisting of exon 1 of PTH2R and exon 20-29 of $A L K$ was identified in an 83-year-old male patient of lung adenocarcinoma. The fusion gene contains exon 1 of $P T H 2 R$ and the entire $A L K$ domain, which may activate $A L K$ signaling continuously to promote tumor progression. This patient responded to crizotinib and alectinib well, but had to discontinue due to AEs. After switching to dose reduction of ceritinib, he kept stable disease till now without insufferable AEs (Figure 3).

ALK-TKIs are extensively used in $A L K$-positive NSCLC, but AEs often limit the clinical application of targeted therapy. In previous meta-analyses, more than $25 \%$ of the ALK-TKI-treated patients reported serious 


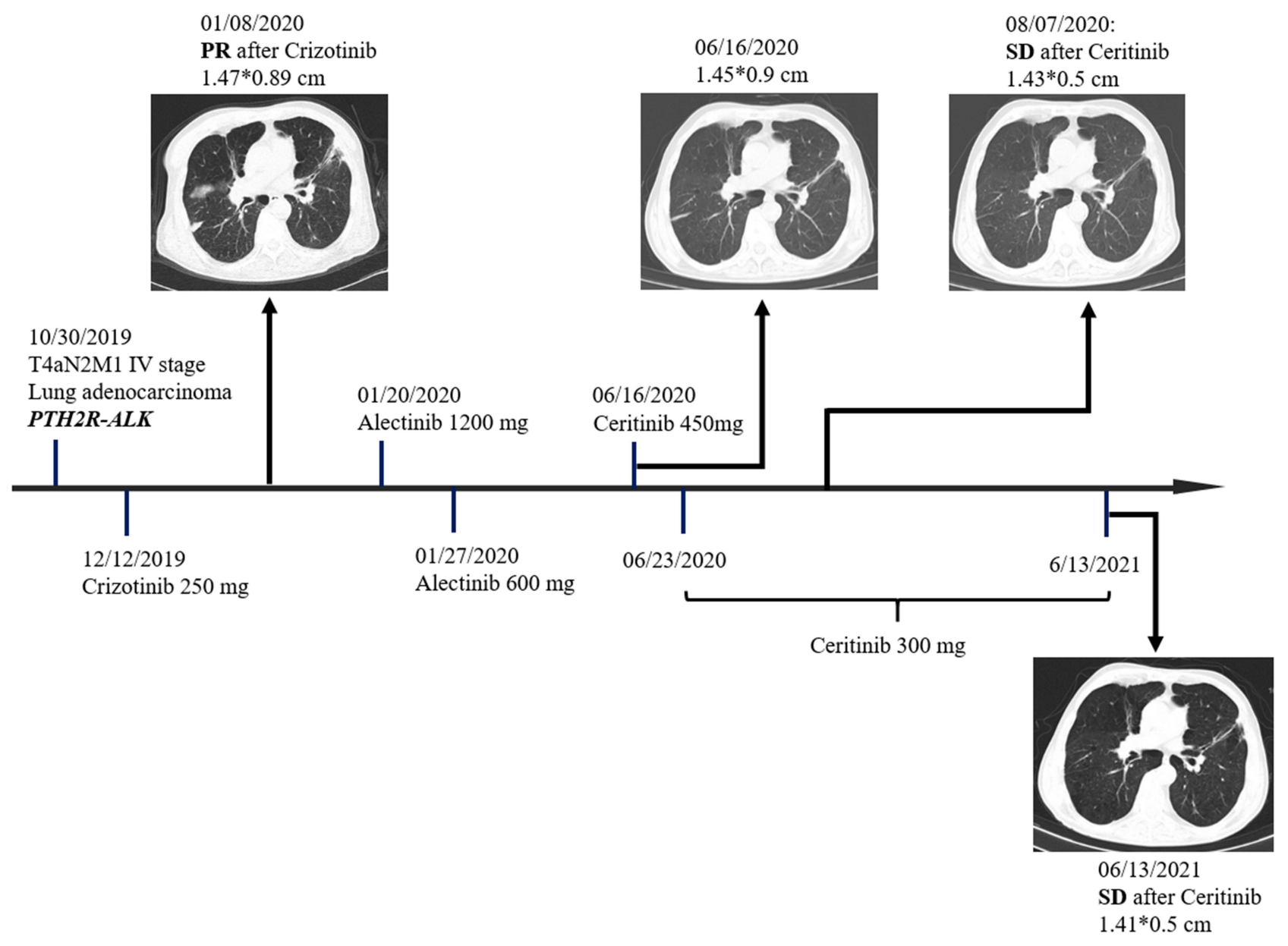

Figure 3 Timeline of the clinical course in this patient.

AEs, ${ }^{12}$ alectinib demonstrated the most favorable safety outcomes, followed by crizotinib and brigatinib, and significant differences were observed in pairwise comparisons. ${ }^{13}$ Due to multiple pathway targets of $A L K$, ALK-TKIs are often associated with a variety of AEs, including gastrointestinal adverse events and hepatotoxicity. Gastrointestinal effects (nausea, vomiting, diarrhea, and constipation) are the most common AEs caused by crizotinib and ceritinib, and have also been reported in alectinib treatment. ${ }^{10}$ Since crizotinib, alectinib and ceritinib are metabolized mainly in the liver, hepatotoxicity is frequently observed when treated with these ALKTKIs. ${ }^{14-16}$ Therefore, it is recommended that patients taking the three agents should be monitored once a month for liver function tests including ALT, AST, $\gamma$-glutamyl transpeptidase $(\gamma-\mathrm{GT})$ and total bilirubin (TBIL). For those patients who show elevations of liver enzymes, particularly during the first 2 months of treatment, tests should be more frequent. Abnormal levels of liver enzymes could be reversible and decreased to baseline in most of patients by dose reduction. Combined with the clinical situation of the patient, dose modification, temporary or permanent drug discontinuation should be considered. ${ }^{14,15}$

The specificity of toxicity profiles among ALK-TKIs may be the reason why this patient showed different tolerance to the three ALK-TKIs. In a real-life cohort, crizotinib, alectinib, and ceritinib treatments displayed similar efficacy but different safety profiles in elderly patients $(\geq 65$ years old). Among the three ALK-TKIs, alectinib was associated with a lower rate of high-grade AEs and a lower treatment discontinuation rate. ${ }^{17}$ In this case, the 83 -year-old man with NSCLC showed better tolerance to ceritinib but not alectinib, which suggests individual variation of patients and response variation of different $A L K$ rearrangement forms.

\section{Conclusion}

To our knowledge, this is the first case in which a novel form of $A L K$ rearrangement $(P T H 2 R-A L K)$ was identified in a lung 
adenocarcinoma patient and his disease has been controlled well after dose reduction of ceritinib. Our findings provide valuable information about how to apply ALK-TKIs in patients with this new form of $A L K$ rearrangement appropriately and suggest that management of AEs would improve the therapeutic outcome of these agents and patients' quality of life.

\section{Ethics Approval and Consent to Participate}

The study was approved by relevant regulatory and independent ethics committee of The Affiliated Kunshan Hospital of Jiangsu University. Written informed consent was obtained from the patient in accordance with the Declaration of Helsinki for the purpose of publication of the present case report and any relevant images.

\section{Funding}

The study was approved by funding of Suzhou Health Key Talent(GSWS2020113).

\section{Disclosure}

Junling Zhang, Xihua Xia, and Mengli Huang were employed by the company Shanghai 3D Medicines Inc. The authors declared no other conflicts of interest.

\section{References}

1. Cao M, Chen W. Epidemiology of lung cancer in China. Thorac Cancer. 2019;10(1):3-7. doi:10.1111/1759-7714.12916

2. Huang H. Anaplastic Lymphoma Kinase (ALK) receptor tyrosine kinase: a catalytic receptor with many faces. Int J Mol Sci. 2018;19 (11):3448. doi:10.3390/ijms19113448

3. Rosas G, Ruiz R, Araujo JM, Pinto JA, Mas L. ALK rearrangements: biology, detection and opportunities of therapy in non-small cell lung cancer. Crit Rev Oncol Hematol. 2019;136:48-55. doi:10.1016/j. critrevonc.2019.02.006

4. Soda M, Choi YL, Enomoto M, et al. Identification of the transforming EML4-ALK fusion gene in non-small-cell lung cancer. Nature. 2007;448(7153):561-566. doi:10.1038/nature05945
5. Du X, Shao Y, Qin HF, Tai YH, Gao HJ. ALK-rearrangement in non-small-cell lung cancer (NSCLC). Thorac Cancer. 2018;9 (4):423-430. doi:10.1111/1759-7714.12613

6. Shaw AT, Yeap BY, Solomon BJ, et al. Effect of crizotinib on overall survival in patients with advanced non-small-cell lung cancer harbouring ALK gene rearrangement: a retrospective analysis. Lancet Oncol. 2011;12(11):1004-1012. doi:10.1016/ S1470-2045(11)70232-7

7. Khan M, Lin J, Liao G, et al. ALK inhibitors in the treatment of ALK positive NSCLC. Front Oncol. 2019;8:557. doi:10.3389/ fonc.2018.00557

8. Yoshida T, Oya Y, Tanaka K, et al. Differential crizotinib response duration among ALK fusion variants in ALK-positive non-small-cell lung cancer. J Clin Oncol. 2016;34(28):3383-3389. doi:10.1200/ JCO.2015.65.8732

9. Shaw AT, Kim TM, Crinò L, et al. Ceritinib versus chemotherapy in patients with ALK-rearranged non-small-cell lung cancer previously given chemotherapy and crizotinib (ASCEND-5): a randomised, controlled, open-label, Phase 3 trial. Lancet Oncol. 2017;18(7):874-886. doi:10.1016/S1470-2045(17)30339-X

10. Rolfo C, Gil-Bazo I, Peters S. Adverse event management in anaplastic lymphoma kinase-positive non-small cell lung cancer. Eur Oncol Haematol. 2015;11:94-99.

11. Zhu Q, Hu H, Weng DS, et al. Pooled safety analyses of ALK-TKI inhibitor in ALK-positive NSCLC. BMC Cancer. 2017;17(1):412. doi:10.1186/s12885-017-3405-3

12. Hou H, Sun D, Liu K, et al. The safety and serious adverse events of approved ALK inhibitors in malignancies: a meta-analysis. Cancer Manag Res. 2019;11:4109-4118. doi:10.2147/CMAR.S190098

13. Chuang $\mathrm{CH}$, Chen HL, Chang HM, et al. Systematic review and network meta-analysis of Anaplastic Lymphoma Kinase (ALK) inhibitors for treatment-naïve ALK-positive lung cancer. Cancers. 2021;13(8):1966. doi:10.3390/cancers 13081966

14. Novartis. Highlights of prescribing information; 2014. Available from: http://www.pharma.us.novartis.com/product/pi/pdf/zykadia. pdf. Accessed December 14, 2021.

15. Pfizer. Highlights of prescribing information; 2014. Available from: http://www.accessdata.fda.gov/drugsatfda_docs/label/2012/ 202570s002lbl.pdf. Accessed December 14, 2021.

16. Gadgeel SM, Gandhi L, Riely GJ, et al. Safety and activity of alectinib against systemic disease and brain metastases in patients with crizotinib-resistant ALK-rearranged non-small-cell lung cancer (AF-002JG): results from the dose-finding portion of a Phase $1 / 2$ study. Lancet Oncol. 2014;15(10):1119-1128. doi:10.1016/S14702045(14)70362-6

17. Bedas A, Peled N, Maimon Rabinovich N, et al. Efficacy and safety of ALK tyrosine kinase inhibitors in elderly patients with advanced ALK-positive non-small cell lung cancer: findings from the real-life cohort. Oncol Res Treat. 2019;42(5):275-282. doi:10.1159/ 000499086
OncoTargets and Therapy

\section{Publish your work in this journal}

OncoTargets and Therapy is an international, peer-reviewed, open access journal focusing on the pathological basis of all cancers, potential targets for therapy and treatment protocols employed to improve the management of cancer patients. The journal also focuses on the impact of management programs and new therapeutic agents and protocols on patient perspectives such as quality of life, adherence and satisfaction. The manuscript management system is completely online and includes a very quick and fair peer-review system, which is all easy to use. Visit http://www.dovepress.com/ testimonials.php to read real quotes from published authors. 